\title{
BJPsych International: its aims and its future
}

\author{
David Skuse ${ }^{\circ}$
}

Professor of Behavioural and Brain Sciences, Division of Population, Policy and Practice, UCL Great Ormond Street Institute of Child Health, London, UK. Email: d.skuse@ucl.ac.uk

A video related to this editorial is available at: https://www.camavailable at: https://www.cam-
bridge.org/core/journals/bjpsychinternational/why-choose bjpsych-internationa

Keywords. Mental Health; evidence-based practice; low and middle income countries; multimedia; education.

First received 25 May 2021 Accepted 25 May 2021

\section{doi:10.1192/bji.2021.37}

(c) The Author(s), 2021. Published by Cambridge University Press on behalf of the Royal College of Psychiatrists. This is an Open Access article, distributed under the terms of the Creative Commons Attribution licence (http://creativecommons.org/ licenses/by/4.0/), which permits licenses/by/4.0/), which permits unrestricted re-use, distribution, and reproduction in any medium provided the original work is properly cited.
Our mission on BJPsych International is simple. First, to promote best practice in the care and treatment of people with mental health problems worldwide. Second, to educate psychiatrists and other mental health professionals about international developments in policy and the delivery of mental health services. We aim to provide a publication platform for authors globally, with a focus on those from low- and middle-income countries who have a message to send about innovations in their country's mental health services that would be of interest to our readership. Our recent success in obtaining a listing on PubMed makes all our articles accessible to a much wider audience and will enhance interest in the journal's unique content. Importantly, the journal, which has a distribution of over 20000 copies, is entirely open access.

\section{Moving with the digital times}

The journal began life as a slim supplement to the British Journal of Psychiatry, published quarterly. Although our printed copy continues to be a quarterly publication, we see the future in terms of a growing online presence. Over the next year or so, we will be substantially increasing the number of articles published annually, of which around one-third will be published online only. There are substantial advantages to authors of online publication, and those articles will be indexed in PubMed in the same way as our printed content. During the COVID crisis, we have received many submissions describing how services around the world are coping with the direct and indirect mental health consequences of the pandemic. Eager to promote that advice, we published all the accepted content online as soon as it had passed peer review. We also publish articles that will eventually appear in the printed journal online; they can be found under the FirstView banner on the journal's website (https://www.cambridge.org/core/journals/bjpsychinternational/firstview).

Many of our readers, especially those from generations who grew up in the digital age, access news and medical education online. We are keen to move with the times, which is why we see our future in terms of a multimedia identity. We will increasingly integrate print and digital content, cognisant of the fact that by expanding our online presence we will drive traffic to the journal and thereby attract a wider audience. In anticipation of this development, we have appointed several Digital Content Editors. Their responsibilities include the promotion of published articles by associating them with podcasts or video material. In the future, we will welcome original video submissions as stand-alone items. The critical evaluation of all multimedia contributions will be an integral part of the publication process. They will be subject to the same scrutiny as printed articles and will be reviewed before publication by experts in the field. We are fortunate to have the support of a team at Cambridge University Press, who are responsible for implementing our digital strategy on the Cambridge Core website and have done so with great efficiency. We encourage you to visit the BJPsych International website (https://www.cambridge.org/core/journals/bjpsych-international) if you have not already done so, where all our published articles, videos and podcasts are available to view free of charge on Vimeo (https://vimeo.com/bjpsych) and SoundCloud (https://soundcloud.com/bjpsych).

\section{Our publishing aims}

We are of course aware that ours is not the only scientific journal that aims to publish on topics relevant to international psychiatry. For that reason, we have carved out a unique identity that it is important for our potential contributors to understand. Despite our wide circulation and readership, we are not accepting articles that are primarily accounts of research projects. There are several reasons why we have decided on that policy. First, as our instructions for authors make clear, articles are a maximum of 1500 words in length. The conventional presentation of original research requires substantially more space. Second, there are several other journals that will consider research-based articles, and their impact factors reflect a readership that is focused on discovering quality publications of that nature. Third, our mission is to provide content that appeals to our readership because it is easy to digest, informative and (when appropriate) entertaining. We give priority to submissions from low- and middle-income countries, especially from regions where original research is difficult or impossible, yet innovative advances in mental health management are competitive in quality, with well-funded centres of excellence. We have had many submissions describing ways 
of working more effectively and efficiently in straitened circumstances. We welcome suggested improvements in the delivery of mental healthcare that are relatively easy to implement and could be adopted widely, at minimal cost.

We aim to provide a forum in which mental health practitioners with a vision that encompasses new approaches to policy and practice can share ideas and learn from one another. These could be disseminated through the @TheBJPsych Twitter account, which provides information about all BJPsych publications. It is also possible to sign up to receive eTOC alerts about our journal - an increasingly popular option. Cambridge University Press makes available a variety of resources with a view to encouraging our authors to promote their articles and engage with our readership. We are strongly committed to rapid publication. It is usually less than a month between a submission to the journal and our first decision about its acceptability. Following final acceptance of an article, it is likely to appear online on FirstView within 30 days.

\section{The journal's structures}

Members of the journal's Editorial Board have been selected to represent each continent, and Board membership is refreshed periodically to ensure that all members participate in lively discussions about strategy and tactics. We place a premium on ideas for content development. Handling Editors, selected from the Editorial Board, manage new submissions. They choose at least two reviewers who are likely to have expertise in the subject matter of the article, although on rare occasions the Handling Editor will act as the second reviewer. Submissions that fall without the scope of the journal, or for other reasons are unlikely to be publishable, are identified before being passed to a Handling Editor; they are pre-screened by the Senior Editorial Team and will not be sent out for review. The final acceptance rate of nonsolicited submissions is currently about $55 \%$.

We have arranged the content of the journal so that it follows a consistent structure. Although most articles are contributed spontaneously, some are commissioned and contribute to the promotion of a particular theme. Our Editorial Board plays an important role in suggesting thematic issues. Individual Board members take responsibility for fostering their favoured theme, which can include subjects as diverse as perinatal mental health and psychiatric aspects of terrorism, by contacting experts in the field. Themes may run through a single issue, or they may spill over into successive issues. Occasionally, we publish a series of articles that encompass a substantial subject, such as Country Profiles or Mental Health Law, over a period of several years. We encourage you to visit our new online collections page (https://www. cambridge.org/core/journals/bjpsych-international/ collections). BJPsych International's editorial team is keen to promote contributions by our junior colleagues, from medical students to trainees in psychiatry. This popular series of articles is named Global Echoes and gives voice to authors who would otherwise find it impossible to publish in an international journal.

\section{Summary}

BJPsych International is a rapidly evolving publication, increasingly focusing on content that takes advantage of resources that are only available online. Our mission is to provide a forum in which practitioners from low- and middle-income countries can share their knowledge and expertise. The journal does not charge a publication fee, it is free to access and is circulated to around 18000 members of the RCPsych. The journal has formerly attracted over 115000 full-text downloads a year and is currently on track to reach 200000 downloads in 2021.

\section{Funding}

This article received no specific grant from any funding agency, commercial or not-for-profit sectors.

\section{Declaration of interest}

D.S. is Editor of BJPsych International.

\section{Supplementary material}

A video is available to view at:

https://www.youtube.com/watch?v=NSyjK8-5bGE. 